\title{
Psychotic-like experiences, polygenic risk scores for schizophrenia, and structural properties of the salience, default mode, and central-executive networks in healthy participants from UK Biobank
}

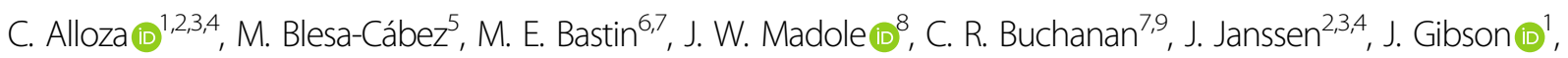
I. J. Deary ${ }^{7}$, E. M. Tucker-Drob ${ }^{8}$, H. C. Whalley $\mathbb{1}^{1}$, C. Arango ${ }^{2,3,4,10}$, A. M. Mclntosh $\mathbb{1}^{1,7}$, S. R. Cox $\mathbb{1}^{7,9}$ and S. M. Lawrie $\mathbb{1}^{1}$

\begin{abstract}
Schizophrenia is a highly heritable disorder with considerable phenotypic heterogeneity. Hallmark psychotic symptoms can be considered as existing on a continuum from non-clinical to clinical populations. Assessing genetic risk and psychotic-like experiences (PLES) in non-clinical populations and their associated neurobiological underpinnings can offer valuable insights into symptom-associated brain mechanisms without the potential confounds of the effects of schizophrenia and its treatment. We leveraged a large population-based cohort (UKBiobank, $N=3875$ ) including information on PLEs (obtained from the Mental Health Questionnaire (MHQ); UKBiobank Category: 144; $\mathrm{N}$ auditory hallucinations $=55, \mathrm{~N}$ visual hallucinations $=79, \mathrm{~N}$ persecutory delusions $=16$, $N$ delusions of reference $=13$ ), polygenic risk scores for schizophrenia $\left(\mathrm{PRS}_{\mathrm{SZ}}\right)$ and multi-modal brain imaging in combination with network neuroscience. Morphometric (cortical thickness, volume) and water diffusion (fractional anisotropy) properties of the regions and pathways belonging to the salience, default-mode, and central-executive networks were computed. We hypothesized that these anatomical concomitants of functional dysconnectivity would be negatively associated with PRS $\mathrm{SZ}$ and PLES. PRS $\mathrm{SZ}$ was significantly associated with a latent measure of cortical thickness across the salience network $(r=-0.069, p=0.010)$ and PLEs showed a number of significant associations, both negative and positive, with properties of the salience and default mode networks (involving the insular cortex, supramarginal gyrus, and pars orbitalis, $p_{\mathrm{FDR}}<0.050$ ); with the cortical thickness of the insula largely mediating the relationship between $\mathrm{PRS}_{\mathrm{Sz}}$ and auditory hallucinations. Generally, these results are consistent with the hypothesis that higher genetic liability for schizophrenia is related to subtle disruptions in brain structure and may predispose to PLES even among healthy participants. In addition, our study suggests that networks engaged during auditory hallucinations show structural associations with PLEs in the general population.
\end{abstract}

Correspondence: C. Alloza (clara.alloza@iisgm.com)

${ }^{1}$ Division of Psychiatry, The University of Edinburgh, Edinburgh, UK 2Department of Child and Adolescent Psychiatry, Institute of Psychiatry and Mental Health, Hospital General Universitario Gregorio Marañón, Madrid, Spain Full list of author information is available at the end of the article.

These authors contributed equally: S. R. Cox, S. M. Lawrie

\section{Introduction}

Schizophrenia is associated with a range of alterations in brain structure and function ${ }^{1-4}$, some of which are related to a family history or specific genetic risk factors. Consistent relationships between delusions, hallucinations, and brain structure and function have, however, proved elusive-potentially because of power issues in relatively small clinical samples and confounds such as 
antipsychotic drug exposure and substance abuse. Psychotic-like experiences (PLEs) of lesser severity are present not only in patients but also in $5-8 \%$ of the general population ${ }^{5}$, and to some extent predict transition to psychiatric disorders among those with higher PLEs ${ }^{6}$; with cohort studies supporting a continuity between subclinical and clinically significant psychotic symptoms ${ }^{7,8}$. A recent study found shared genetic aetiology between PLEs and several psychiatric and neurodevelopmental disorders, indicating that PLEs may be related to a general risk for mental health disorders ${ }^{9}$. Studies of the relationship between PLEs and brain imaging metrics have, however, been scarce and characterized by small sample sizes (with $N$ ranging from 25 for auditory hallucinations to 76 for any PLEs ${ }^{10-13}$ ). Functionally, PLEs have been associated with altered brain dynamics, in particular with default-mode hypoconnectivity ${ }^{11,14}$. Nonetheless, such evidence validates the study of the pathophysiology of these clinical phenotypes in nonclinical populations ${ }^{15}$, with large studies from the general population also offering increased power to detect such effects.

Aberrant functioning and organization of the salience network, default mode network (DMN), and centralexecutive network (CEN) are core features of several psychiatric and neurological disorders ${ }^{16}$; with patients with a diagnosis of schizophrenia showing structural and functional impairments in all three networks ${ }^{17}$. The salience network includes the insula and anterior cingulate cortex, and is involved in the identification of biological and behaviourally relevant stimuli and the subsequent coordination of neural resources to guide flexible behaviour $^{18,19}$. Aberrant intrinsic functional connectivity of the salience network has been observed in schizophrenia $^{20,21}$ and in individuals at clinical high risk for psychosis $^{22}$, and is posited to underlie persecutory delusions in particular ${ }^{23}$. The DMN is a distributed system of fronto-temporal-parietal cortex that is activated during passive cognitive states and deactivates during several cognitive tasks ${ }^{24}$. In schizophrenia, the DMN is overactive with significant correlations between the activity of subregions of the DMN and positive symptoms ${ }^{25}$; although the evidence is somewhat inconsistent ${ }^{25-27}$. The CEN is a frontoparietal system coactivating the dorsolateral prefrontal cortex and the posterior parietal cortex during several cognitive tasks ${ }^{28}$. Increased functional connectivity between the DMN and CEN has been linked to hallucinations in patients with schizophrenia ${ }^{20}$.

Schizophrenia is highly polygenic, with many common alleles of small effect, and increasing numbers of genomewide significant loci have been identified as sample sizes increase ${ }^{29-31}$. Summary statistics from large-scale genome-wide association study (GWAS) allow the degree of genetic liability for a heritable trait to be estimated in healthy subjects outside the population in which the original GWAS was conducted ${ }^{32,33}$. Only a small number of studies have analyzed the relationship between polygenic risk score for schizophrenia $\left(\mathrm{PRS}_{\mathrm{SZ}}\right)$ and neuroimaging biomarkers in healthy samples ${ }^{32-37}$ but some of these associations map on to regions likely to be involved in the generation of psychotic symptoms and PLEs.

Thus, in this study we investigated how $\mathrm{PRS}_{\mathrm{SZ}}$ relates to neuroanatomical properties of the salience network, DMN, and CEN, and thence to PLEs; in addition to formally testing whether the association between $\mathrm{PRS}_{\mathrm{SZ}}$ and PLEs was mediated by brain structure. We computed water diffusion magnetic resonance imaging (MRI) parameters of white matter tracts (using fractional anisotropy; FA), cortical thickness (CT), and grey matter volume (GMV) of the regions involved in these networks in a large sample of healthy participants from UKBiobank in whom any such associations would not be confounded by illness-associated factors. We employed a novel approach based on ROI-ROI analysis (derived from connectome processing) which extends our previous a priori network selection methods ${ }^{38,39}$, allowing a much finer-grained network approach than using other techniques which quantify white matter connectivity without direct subjectspecific linkage to cortical or subcortical regions. In addition, previous studies have suggested that schizophrenia may be accompanied by accelerated ageing ${ }^{40}$, indicating for instance, significant declines in white matter coherence more than twice that of age-matched controls $^{41}$. Therefore, to examine possible accelerated brain ageing in brain structure, we included an interaction term between age at MRI scanning and $\mathrm{PRS}_{\mathrm{SZ}}$ in all analyses.

\section{Methods \\ Participants}

UKBiobank (http://www.ukbiobank.ac.uk/) comprises around 500,000 community-dwelling participants recruited from across the United Kingdom of Great Britain and Northern Ireland between 2006 and 2014. A subset of the participants who were part of the initial recruitment attended for head MRI scanning at an average of around 4 years after the initial visit (all data presented in this analysis were collected on the same scanner). The current study uses the $5 \mathrm{k}$ neuroimaging data release (Supplementary Fig. 1). UKBiobank received ethical approval from the Research Ethics Committee (reference 11/NW/ 0382). Those participants who had been admitted to a hospital with a diagnosis of schizophrenia or bipolar disorder with psychotic symptoms were excluded from our analysis. In order to comprehensibly study the PLEs phenotype, additional analyses comprised: (1) the exclusion of participants who had any psychiatric-related admissions (diagnoses in Supplementary Table 1) and (2) the inclusion of the whole sample $(N=3875)$. The 
present analyses were conducted as part of UKBiobank application 16,124, linked to 4844 and 10,279. All participants provided informed consent (http://biobank.ctsu. ox.ac.uk/crystal/field.cgi?id=200).

\section{Polygenic risk score calculation}

The details of the array design, genotyping, quality control, and imputation have been described previously ${ }^{42}$. Quality control included removal of participants based on missingness, relatedness, gender mismatch non-British ancestry, and participants based upon overlap in Psychiatric Genomics Consortium (PGC) prediction samples and schizophrenia status. Polygenic profiles were created for schizophrenia in all the genotyped participants from UKBiobank using PRSice ${ }^{43}$. PRSice calculates the sum of alleles associated with the phenotype of interest across many genetic loci, weighted by their effect sizes estimated from a genome-wide association study of that phenotype in an independent sample. These effect sizes have been estimated previously in GWAS for schizophrenia (PGC-SCZ, https://www.med.unc.edu/pgc/pgc-workgroups; 36,989 cases, 113,075 control subjects).

Before creating the scores, single nucleotide polymorphisms (SNPs) with a minor allele frequency $<1 \%$ were removed and clumping was used to obtain SNPs in linkage equilibrium with an $r^{2}<0.25$ within a $200 \mathrm{bp}$ window. Five scores were created for each individual using SNPs selected according to the significance of their association with the phenotype at nominal $p$-value thresholds of 0.01, 0.05, 0.1, 0.5, and 1.0 (all SNPs). Our primary analyses used scores generated from a list of SNPs with a GWAS training set of $p \leq 0.1$, as this threshold was shown to explain the most phenotypic variance in the discovery cohort ${ }^{31}$ (results for 0.5 and 1.0 thresholds are presented in Supplementary Results 1). Fifteen multidimensional scaling factors (estimated from SNP data) were entered into all models as additional "nuisance" covariates to control for population stratification, along with age and genotyping array.

\section{Psychotic-like experiences information}

Lifetime PLEs were taken from a Mental Health Questionnaire (MHQ; UKBiobank Category: 144) and was sent to all participants who provided an email address from July 2016 to July 2017. Responses to the following questions were dichotomized: "Did you ever believe that there was an unjust plot going on to harm you or to have people follow you, and which your family and friends did not believe existed?", "Did you ever believe that a strange force was trying to communicate directly with you by sending special signs or signals that you could understand but that no one else could understand (for example through the radio or television)?", "Did you ever hear things that other people said did not exist, like strange voices coming from inside your head talking to you or about you, or voices coming out of the air when there was no one around?", and "Did you ever see something that wasn't really there that other people could not see?". We categorized these PLEs as persecutory delusions, delusions of reference, and auditory and visual hallucinations, respectively. This questionnaire explicitly indicated not to include those instances when the participant was dreaming, half-asleep, or under the influences of alcohol or drugs. Moreover, level of distress in relation to PLEs was defined as "Not distressing at all, it was a positive experience", "Not distressing, a neutral experience", "A bit distressing", "Quite distressing", and "Very distressing", and was coded as a continuous variable (ranging from -1 to 3 , in order of increasing distress). Participants that responded "do not know" or "prefer not to answer" were excluded from analyses in all cases.

\section{Imaging analysis}

Full details of the image acquisition and processing can be found on the UKBiobank website (http://biobank.ctsu. ox.ac.uk/crystal/refer.cgi?id=2367), Brain Imaging Documentation (http://biobank.ctsu.ox.ac.uk/crystal/refer. cgi?id=1977), and in ref. ${ }^{44}$. More information regarding scan acquisition and image processing can be found in Supplementary Methods 1 and Supplementary Table 2 for a list of abbreviations of each node. Network nodes were selected based on previous studies, for the salience network we selected the following regions: thalamus, amygdala, ventral diencephalon, caudal anterior cingulate, and insula ${ }^{18,45}$; for the DMN: lateral orbitofrontal, medial orbito frontal, middle temporal, pars orbitalis, posterior cingulate, and supramarginal ${ }^{28,46}$; and for the CEN: caudal middle frontal, inferior parietal, rostral middle frontal, superior frontal, and superior parietal ${ }^{45,47,48}$. Figure 1 shows the nodes and white matter pathways selected for each of the networks studied here. For the present study, data available for participants who were unrelated, survived the quality control process and had full imaging data available, is provided in Table 1.

\section{Network construction}

For each subject, two networks were constructed: the number of streamline (NOS) network that was created using the number of streamlines connecting each pair of the 85 ROI (network node) pairs from the default FreeSurfer cortical ${ }^{49}$ and subcortical regions (Desikan-Killiany atlas, see Supplementary Table 2 for a list of abbreviations of each node); and the FA-weighted network that was constructed by recording the mean FA value along streamlines. The endpoint of a streamline was considered to be the first grey matter ROI encountered when tracking from the seed location. In order to reduce the number of spurious connections derived from 


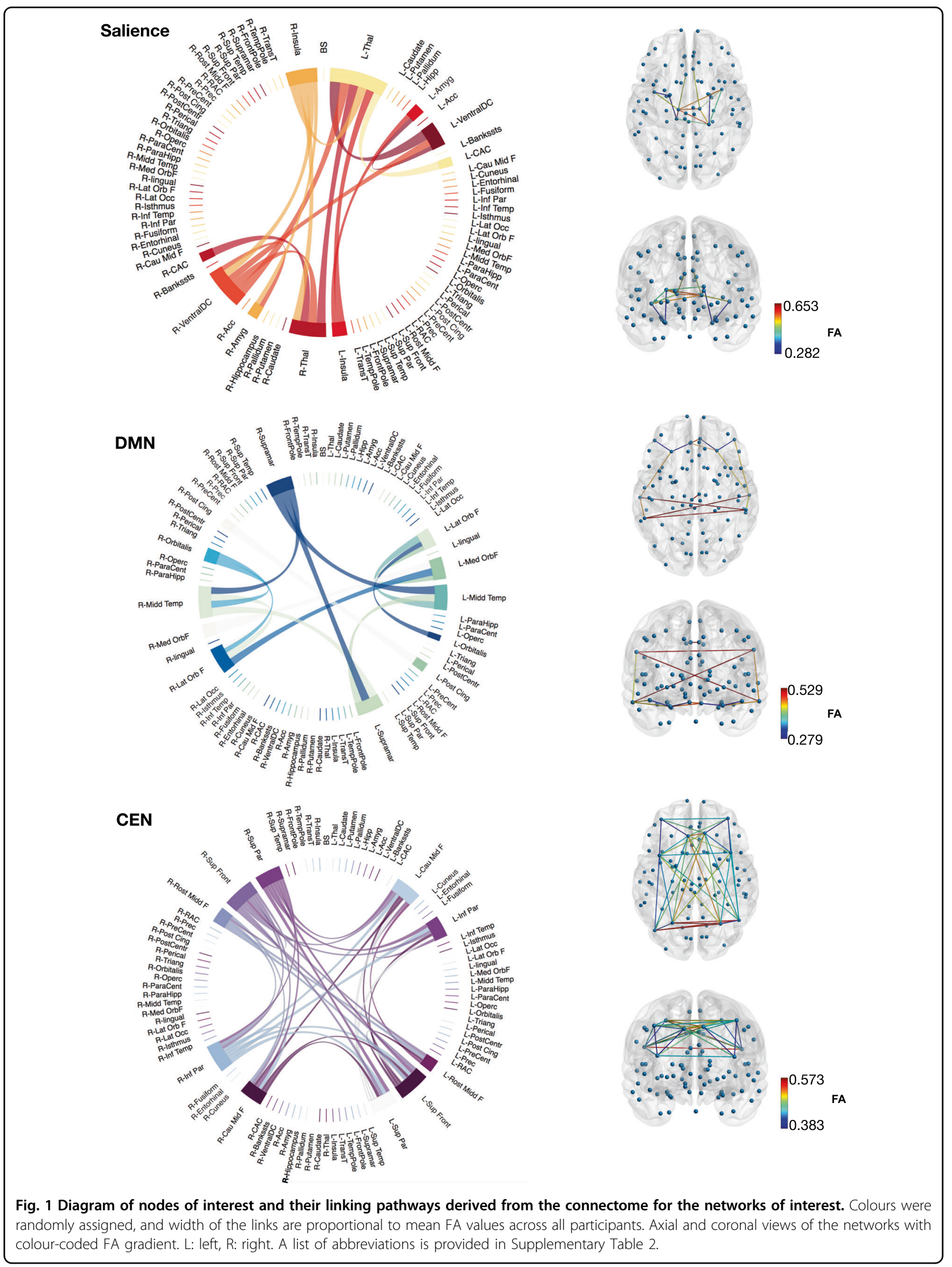


Table 1 Participants characteristics.

\begin{tabular}{|c|c|c|c|c|}
\hline & Variable & Units & Descriptor & Valid $\Lambda$ \\
\hline \multirow[t]{2}{*}{ Demographics } & Age & Years (SD) & $62.18(7.57)$ & 3875 \\
\hline & Sex & $F, \%$ females & $2032(52.44)$ & 3875 \\
\hline \multirow[t]{5}{*}{ PLEs } & Presence of at least one & Yes (\% yes) & $134(4.75 \%)^{\mathrm{a}}$ & 2819 \\
\hline & Persecutory delusions & Yes (\% yes) & $16(0.57 \%)^{a}$ & $13: 130^{b}$ \\
\hline & Delusions of reference & Yes (\% yes) & $13(0.46 \%)^{a}$ & $11: 110^{b}$ \\
\hline & Auditory hallucinations & Yes (\% yes) & $55(1.95 \%)^{a}$ & $51: 510^{b}$ \\
\hline & Visual hallucinations & Yes (\% yes) & $79(2.80 \%)^{a}$ & $70: 700^{b}$ \\
\hline \multirow[t]{3}{*}{ Brain MRI } & Cortical thickness & mm Mean (SD) & $2.40(0.10)$ & 3566 \\
\hline & Grey matter volume & $\mathrm{mm}^{3}$ Mean (SD) & $620,504.60(55,712.52)$ & 3868 \\
\hline & Mean FA & Units (SD) & $0.46(0.02)$ & 3174 \\
\hline
\end{tabular}

Note: complete data after quality control and exclusion criteria. SD = standard deviation. Brain MRI volumes are uncorrected for head size.

aPercentages and rates were calculated exclusively on participants answering either "Yes" or "No" to PLEs $(N=2819)$.

bDue to the relatively low frequencies of participants answering "Yes" to any PLEs, these participants were matched to those answering "No" using a ratio of 1:10 for each PLE for complete data (see "Methods" for more detail). Note that these sample sizes may vary for the different brain structural properties analyzed.

probabilistic tractography, we applied a consistencybased threshold to the NOS matrices using the numbers of streamlines connecting all 85 ROI and preserving exclusively the top $30 \%$ white matter tracts that were most consistent across subjects ${ }^{50}$. This mask was then applied to the FA-weighted connectivity matrices. For each FA-weighted connectivity matrix for the thresholded network, the salience, DMN and CEN masks were applied based on our bilateral nodes of interest. Mean FA was computed using only the non-zero matrix elements.

\section{Statistical analyses}

We undertook an a priori network-of-interest (NOI) approach, based on the literature cited above.

\section{Linear regressions for individual network components}

Initially, we conducted linear regressions between $\mathrm{PRS}_{\mathrm{SZ}}$ and each node (CT, GMV) and edge (FA) within each of the selected NOIs. Within each model, each morphometric measure was set as the dependent variable, $\mathrm{PRS}_{\mathrm{SZ}}$ as the independent variable, controlling for age, sex, and the interaction between age and $\mathrm{PRS}_{\mathrm{SZ}}$.

\section{Network analyses}

Next, we aimed to test whether associations with $\mathrm{PRS}_{\mathrm{SZ}}$ were best represented in the data at the network-general level (common pathway), or whether there were additional unique associations with specific network components (common + independent pathways analysis), or whether the associations were simply best described by independent pathways ${ }^{51}$. We did so in a structural equation modelling (SEM) framework. First, we fitted measurement models (i.e., models that relate the latent factor to its manifest variables) to ascertain the degree to which we could describe overall network integrity at the network level; this initial measurement model was a confirmatory factor analysis in which we tried to estimate a single latent construct of global NOI by incorporating altogether grey and white matter metrics pertaining to all nodes within a NOI. However, these models all exhibited poor fit to the data, and the latent measures of the grey and white matter did not correlate significantly.

Common model analysis Thus, we opted to construct three measurement models for each NOI in which a latent factor was indicated by all network components separately: CT (derived from the bilateral cortical nodes), GMV (including all bilateral cortical and subcortical nodes), or white matter FA (derived from all pathways connecting nodes within each NOI). These measurement models all fitted the data well except for FA in the DMN and CEN which did not achieve acceptable model fit statistics and thus, were not included in these analyses.

For each model, we then tested associations between $\mathrm{PRS}_{\mathrm{SZ}}$, age, the interaction $\mathrm{PRS}_{\mathrm{SZ}} \times$ age, and MRI parameters. We did so by fitting multiple indicators, multiple causes (MIMIC) model $^{52}$; Fig. 2a shows a simplified diagram of the SEM framework (see example of code used in Supplementary Methods 2). Within the model, each brain imaging measure was adjusted at the manifest level for sex and either whole brain average $\mathrm{CT}$, whole brain total GMV, or whole brain mean FA, while $\mathrm{PRS}_{\mathrm{SZ}}$ was adjusted for sex, population stratification components, and genotyping array. Model fits were assessed using the following indices and cut-offs: Comparative Fit Index (CFI), Root Mean Square Error of Approximation (RMSEA), and Standardized Root Mean Square Residual 
A MIMIC model

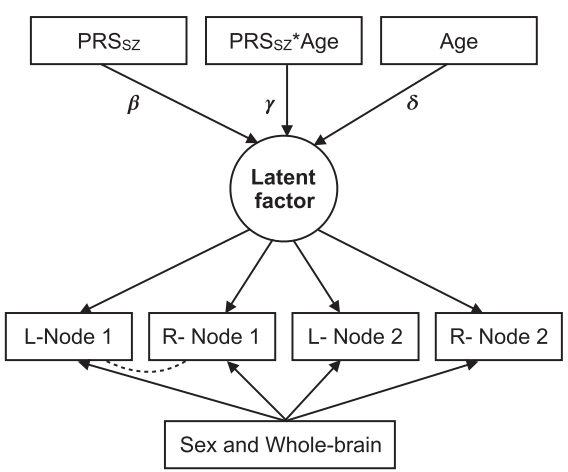

B Mediation model

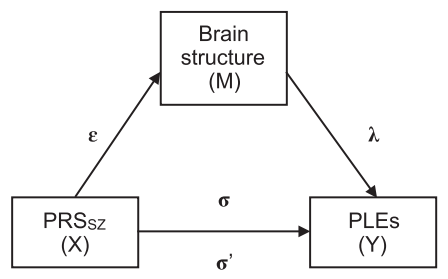

Fig. 2 Diagrams of structural equation models (SEM). a Multiple indicators, multiple causes (MIMIC) model $\left.\right|^{52}$ for neurostructural properties of each network. A separate model was applied to FA, grey matter thickness (CT), and grey matter volume (GMV). From each individual bilateral node (L: left; R: right) or pathway, a latent score was calculated for FA, CT, GMV controlling for sex and whole-brain structural properties at the manifest level (i.e., whole brain FA/CT/GMV). Relation between FA/CT/GMV and PRS $\mathrm{Sz}$ is indicated by path $\beta$; path $\gamma$ represents the association between the interaction of age and PRS SZ and FA/CT/GM factors; path $\delta$ represents the association between age and the latent factor. PRS SZ $_{\text {W }}$ was corrected for sex and population stratification (paths not shown). The dashed line represents a possible residual correlation between nodes. b Path diagram of mediation model, where the $\varepsilon$ coefficient represents the regression of $X$ on $M, \lambda$ coefficient the regression of $M$ on $Y$, and $\sigma$ coefficient the direct path of $X$ on $Y$. The product of the $\varepsilon$ and $\lambda$ coefficients describes the indirect path of $X$ on $Y$ through $M\left(\sigma^{\prime}\right)$.

(SRMR). We allowed for certain residual correlations between manifest variables in those cases in which their addition improved the model fit significantly. SEM analyses were conducted using the package "lavaan" 53 in $\mathrm{R}$ with full-information maximum likelihood estimation to use all data available.

MIMIC common + independent pathways analysis To assess whether associations between $\mathrm{PRS}_{\mathrm{SZ}}$ and latent constructs of network integrity were best characterized at the network-general level, or whether there were additional associations with particular brain regions beyond this, we ran a common plus independent pathways analysis $^{51}$ (see details in Supplementary Methods 3).

\section{PLES}

Due to the low percentage of participants who answer "Yes" to experiencing PLEs (see Table 1), we first matched those participants to those who answered "No" by age and sex with a ratio 1:10 using the function "match.it", a propensity score method from the "MatchIt" package ${ }^{54}$. Linear regressions were then calculated independently for each network metric (including all network nodes or edges) with each PLEs as the response variable, covarying for either whole brain total volume (for node's volume; to control for differences in whole-brain volume across participants), average CT (for node's CT; to control for differences in whole-brain CT across participants) or whole brain mean FA (for edges; to control for differences in whole-brain FA across participants), age at MRI and sex. Therefore, correction for multiple comparisons was performed within each network metric and for each PLE. Raincloud plots ${ }^{55}$ were used to plot group differences between participants with PLEs and their matched controls.

\section{Mediation analyses}

We further sought to formally test whether the association between PRS $_{\mathrm{SZ}}$ and PLEs was mediated by brain structure (at the manifest level). We did this in the form of a mediation model using the "lavaan" package ${ }^{53}$ with fullinformation maximum likelihood estimation, confidence intervals were reported (CI; constructed using 1000 bootstraps). This analysis was performed only in those cases where the path between brain structure $(M)$ and PLEs ( $\mathrm{Y}$ ) was significant $\left(p_{\mathrm{FDR}}<0.050\right)$; mediation was observed when the change from $\sigma$ to $\sigma^{\prime}$ was statistically significant (confidence intervals did not include zero). Within the model, each brain region was adjusted for age, sex, and either average CT (CT analysis) or whole brain total volume (GMV analysis). Figure $2 \mathrm{~b}$ shows a simplified diagram of the mediation framework.

\section{Additional analyses}

In order to determine whether the signal could be driven by any complication associated with any psychiatric disorder beyond a diagnosis of psychosis (i.e., distress, dysfunction, co-morbidities, medication, unhealthy lifestyles, etc.), we also performed an analysis that excluded all participants with any psychiatric diagnoses from the PLEs analysis (Supplementary Results 2). Due to the possible clinical significance of level of distress in relation to PLEs, we first computed logistic regressions with PLEs 
as the response variable and level of distress, age, and sex as predictor variables (using the "lm" function from the "stats v3.6.2" package). For those significant associations, we tested whether the level of distress caused by PLEs could be mediating the relationship between brain structure and PLEs (Supplementary Results 3). We also tested the hypothesis that increased number of PLEs (i.e., individual sum of these four types of PLEs) was associated with a higher level of distress ${ }^{56}$ (Supplementary Results 4 ).

All analyses were performed in $\mathrm{R}$ (https://www.rproject.org) and standardized betas were reported throughout. For each section/model of statistical analysis, significance $(p)$ values $(\alpha=0.050)$ were corrected for multiple comparisons using false discovery rate $(\mathrm{FDR})^{57}$ (using the "p.adjust" function from the "podkat" package).

\section{Results}

Participant characteristics are presented in Table 1, and PLEs prevalence in Supplementary Table 3. Mean values for CT and GMV for each node (according to the Desikan-Killiany atlas ${ }^{49}$ ) are shown in Supplementary Fig. 2. All SEM models showed acceptable fit to the data (fit indices are shown in Supplementary Table 4).

\section{$\mathrm{PRS}_{\mathrm{SZ}}$ analyses}

\section{Salience network}

Linear regressions for individual network components There were no significant associations between cortical and subcortical GMV and $\mathrm{PRS}_{\mathrm{SZ}}\left(p_{\mathrm{FDR}}>0.050\right)$. For CT, nominally significant negative associations were found between the right and left insula and $\mathrm{PRS}_{\mathrm{SZ}}\left(\beta=-0.046, \mathrm{SE}=0.019, p_{\mathrm{FDR}}=\right.$ $0.050, \beta=-0.039, \mathrm{SE}=0.018, p_{\mathrm{FDR}}=0.065$, respectively). Interactions between age and $\mathrm{PRS}_{\mathrm{SZ}}$ were significant for $\mathrm{CT}$ of the right caudal anterior cingulate (CAC) $(\beta=-0.054$, $\left.\mathrm{SE}=0.021, p_{\mathrm{FDR}}=0.040\right)$. There were no significant associations between FA and $\mathrm{PRS}_{\mathrm{SZ}}\left(p_{\mathrm{FDR}}>0.050\right)$.

MIMIC common + independent pathways analysis The association between the latent factor for GMV and $\mathrm{PRS}_{\mathrm{SZ}}$ was not significant $(r=-0.026, \mathrm{SE}=0.014, p=$ 0.242). Allowing for a direct effect of $\mathrm{PRS}_{\mathrm{SZ}}$ on left thalamus GMV significantly improved model fit $\left(\mathrm{x}^{2}[1]>\right.$ 4.917, $p<0.028$; independent pathway estimates: $\beta=$ $0.017, p=0.032$ ). A direct effect of right CAC GMV on $\mathrm{PRS}_{\mathrm{SZ}} \times$ age $(\beta=-0.035, p=0.088)-$ the second independent pathway added-significantly improved model fit $\left(\chi^{2}[1]=5.893, p=0.015\right)$. Results are shown in Fig. 3a. In addition to the association between $\mathrm{PRS}_{\mathrm{SZ}}$ and the latent factor of CT $(r=-0.069, \mathrm{SE}=0.015, p=0.010)$, an independent pathway emerged from $\mathrm{PRS}_{\mathrm{SZ}} \times$ age to right CAC CT $(\beta=-0.043, p=0.031)$, which improved the model fit $\left(\chi^{2}[1]=4.606, p=0.030\right.$; Fig. 3b). There was no significant association between the latent factor for FA and either $\mathrm{PRS}_{\mathrm{SZ}}$ or $\mathrm{PRS}_{\mathrm{SZ}} \times$ age interaction $(p>0.050)$.

\section{Default mode network}

Linear regressions for individual network components There were no significant associations between GMV, CT or FA and $\mathrm{PRS}_{\mathrm{SZ}}$ or the interaction between $\mathrm{PRS}_{\mathrm{SZ}} \times$ age $\left(p_{\mathrm{FDR}}>0.050\right)$.

MIMIC common +independent pathways analysis The associations between the latent factors for GMV and $\mathrm{CT}$ and $\mathrm{PRS}_{\mathrm{SZ}}$ were not significant $\left(r_{\mathrm{GMV}}=-0.040\right.$, $\mathrm{SE}=0.012, \quad p=0.111 ; \quad r_{\mathrm{CT}}=-0.028, \quad \mathrm{SE}=0.015, \quad p=$ $0.273)$. An independent pathway emerged from $\mathrm{PRS}_{\mathrm{SZ}} \times$ age to right supramarginal CT $\left(\chi^{2}[1]>5.705, p<0.018\right.$; independent pathway estimate: $\beta=-0.035, p=0.008$ ). Interactions between $\mathrm{PRS}_{\mathrm{SZ}} \times$ age were not significantly associated with the latent constructs $(p>0.050)$.

\section{Central executive network}

Linear regressions for individual network components No significant associations were found between the GMV, $\mathrm{CT}$ or FA and $\mathrm{PRS}_{\mathrm{SZ}}$, or the interaction between $\mathrm{PRS}_{\mathrm{SZ}} \times$ age $(p>0.050)$.

MIMIC common + independent pathways analysis The associations between the latent factors for grey matter and $\mathrm{PRS}_{\mathrm{SZ}}$ were not significant $(p>0.050)$. For GMV, allowing for a direct effect of $\mathrm{PRS}_{\mathrm{SZ}}$ on right inferior parietal GMV $(\beta=0.039, p=0.004)$ resulted in significant improvement in model fit $\left(\chi^{2}[1]>7.741, p<\right.$ 0.006). For $C T$, an independent pathway emerged from $\mathrm{PRS}_{\mathrm{SZ}} \times$ age to right superior frontal CT $\left(\mathrm{X}^{2}[1]>7.512\right.$, $p<0.007$; independent pathway estimate: $\beta=0.028, p=$ $0.002)$ and right inferior parietal $\mathrm{CT}\left(\mathrm{x}^{2}[1]>4.314, p<\right.$ 0.039 ; independent pathway estimate: $\beta=-0.024, p=$ 0.034). Interactions between $\mathrm{PRS}_{\mathrm{SZ}} \times$ age were not significantly associated with any latent construct $\left(p_{\mathrm{FDR}}>\right.$ 0.050).

\section{Psychotic-like experiences}

Table 1 shows the frequencies and percentages of respondents with PLEs, Supplementary Table 3 shows sample characteristics for participants reporting PLEs versus non-PLEs. Figure 4a shows raincloud plots representing differences in CT and GMV between participants reporting PLEs and their matched controls.

Within the salience network, significant negative associations were found between the $\mathrm{CT}$ of the right and left insula and auditory hallucinations $\left(N_{\text {cases }}=51, N_{\text {controls }}=\right.$ $510 ; \beta=-0.114, \quad \mathrm{SE}=0.035, \quad p_{\mathrm{FDR}}=0.004$ and $\beta=$ $-0.083, \mathrm{SE}=0.036, p_{\mathrm{FDR}}=0.045$, respectively). Associations between total number of PLEs and CT/GMV of salience network nodes were all non-significant. 




B

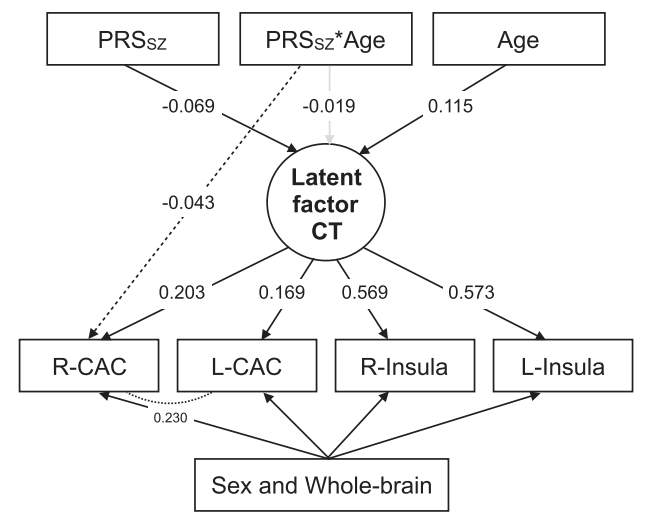

Fig. 3 Diagrams of MIMIC and independent pathway models of the salience network. a Common + independent pathways model for latent factor of grey matter volume. $\mathbf{b}$ Common + independent pathways model for latent factor of grey matter cortical thickness. Dashed lines represent independent pathways while dotted lines residual correlations between nodes. Black lines represent statistically significant pathways $(p<0.050)$ while grey lines non-significant pathways $(p>0.050)$.

For the default mode network, a significant negative association was found between the GMV of the right supramarginal gyrus and delusions of reference $\left(N_{\text {cases }}=\right.$ $\left.11, N_{\text {controls }}=110 ; \beta=-0.195, \mathrm{SE}=0.061, p_{\mathrm{FDR}}=0.022\right)$ and between persecutory delusions and GMV of the left pars orbitalis $\left(N_{\text {cases }}=12, N_{\text {controls }}=120 ; \beta=0.219, \mathrm{SE}=\right.$ $\left.0.069, p_{\mathrm{FDR}}=0.021\right)$. For persecutory delusions, there was a positive association with the $\mathrm{CT}$ of the right supramarginal gyrus $\left(N_{\text {cases }}=13, N_{\text {controls }}=130 ; \beta=0.177\right.$, $\left.\mathrm{SE}=0.048, p_{\mathrm{FDR}}=0.003\right)$. We did not find any significant association between total number of PLEs and GMV/CT of nodes. No significant associations were found between CEN's nodes and PLEs $\left(p_{\mathrm{FDR}}>0.050\right)$. The overall PLE findings are summarized in Supplementary Table 5.

\section{Mediation analyses}

The mediation models using $\mathrm{PRS}_{\mathrm{SZ}}$ and mediators met the criteria for a close-fitting model (RMSEA $=0, \mathrm{CFI}=$ 1 , SRMR < 0.05). We examined the hypothesis that higher $\mathrm{PRS}_{\mathrm{SZ}}$ was associated with symptom severity via the brain structures significantly associated with PLEs in the salience and DMN. First, we tested the association between $\mathrm{PRS}_{\mathrm{SZ}}$ and total number of PLEs in the whole UKBiobank sample, independently of diagnosis $\left(N_{\text {PRS }}=302,575\right.$, $N_{\text {PLEs }}=157,305$, with $N=7803$ answering "Yes" to experiencing at least one PLEs). However, we did not find any significant association $(\beta=0.020, \mathrm{SE}=0.018, p=$ 0.283 ). We found that the linear association between $\mathrm{PRS}_{\mathrm{SZ}}$ and auditory hallucinations was significantly mediated by $\mathrm{CT}$ of the right insula (from $\sigma=0.023$ to $\sigma^{\prime}=0.004$, CI $[0.020,0.127]$ with the right insular cortex mediating $82.6 \%$ of the association between $\mathrm{PRS}_{\mathrm{SZ}}$ and auditory hallucinations, Fig. 5). These results were consistent across all PRS $_{\mathrm{SZ}}$ thresholds (Supplementary Results 5). No significant mediations were observed for any other ROIs.

\section{Discussion}

The present study aimed to investigate different structural properties of the salience, DMN, and CEN networks in 

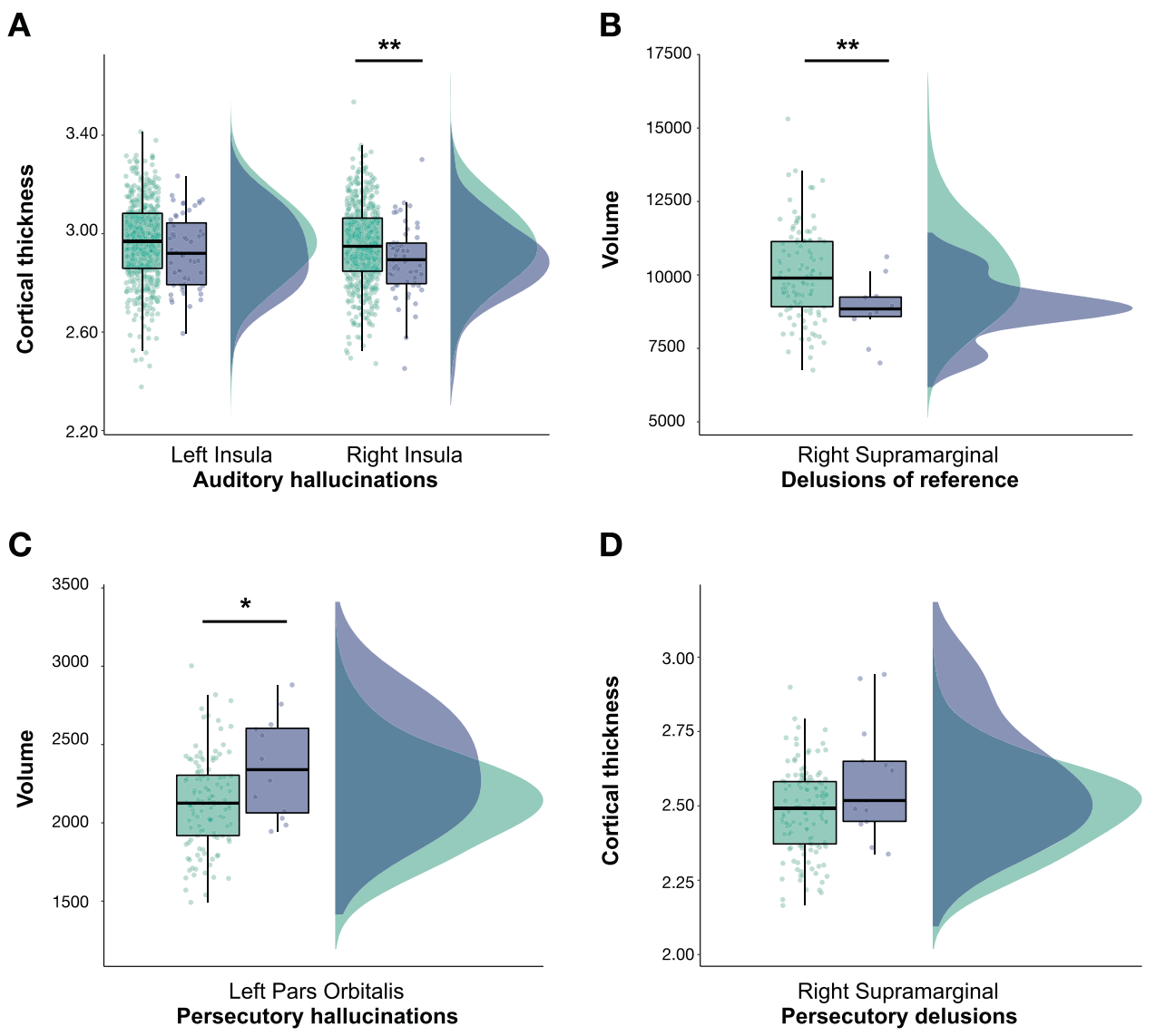

Fig. 4 Raincloud plots representing differences in cortical thickness (CT) and volume (GMV) between participants reporting PLEs (blue) and matched controls (green). a Bilateral $C T$ of the insula and auditory hallucinations. b GMV of right supramarginal gyrus and delusions of reference. $\mathbf{c}$ GMV of the left pars orbitalis and persecutory delusions. $\mathbf{d} C T$ of the right supramarginal gyrus and persecutory delusions. Note: $\mathrm{CT}$ is measured in $\mathrm{cm}$ and $\mathrm{GMV}$ in $\mathrm{cm}^{3}$. Asterisks represent significant differences between groups $\left({ }^{*} p<0.05,{ }^{* *} p<0.01\right)$.

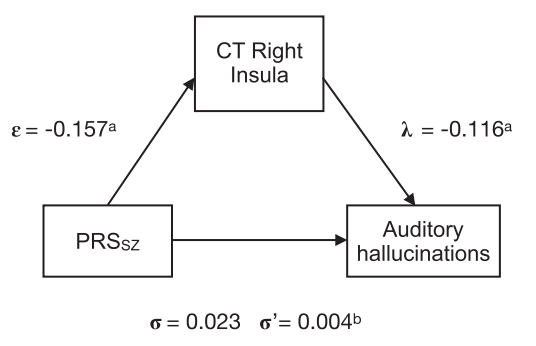

Fig. 5 Path diagram of the mediation model, where the $\varepsilon$ coefficient represents the coefficient of regressions of $\mathrm{PRS}_{\mathrm{SZ}}$ on cortical thickness (CT) of the right insula, and $\lambda$ the coefficient of the regression of $\mathrm{CT}$ of the right insula on auditory hallucinations, and $\sigma$ coefficient of the direct path of $\mathrm{PRS}_{\mathrm{Sz}}$ and auditory hallucinations. Coefficient of $\sigma^{\prime}$ represents the indirect path of $\mathrm{PRS}_{\mathrm{Sz}}$ on auditory hallucinations through $\mathrm{CT}$ of the right insula. Note: Superscript a represents $p$ values $<0.01$ and superscript b refers to confidence intervals not including zero.

relation to $\mathrm{PRS}_{\mathrm{SZ}}$ and PLEs in a large population-based cohort of healthy participants. This is, to our knowledge, the first study to investigate these polygenic-MRI associations using the structural properties of brain networks from a connectome perspective, where the properties of estimated connections that directly link distal cortical and subcortical regions in each individual are examined.

At the node level, we found nominally significant associations between thinner right and left insular cortices and higher $\mathrm{PRS}_{\mathrm{SZ}}$. At the network level we found that higher PRS $\mathrm{SZ}_{\mathrm{SZ}}$ was associated with reduced CT across the salience network, with some specific associations between $\mathrm{PRS}_{\mathrm{SZ}}$ and individual brain properties emerging. The findings reported here indicate both global network and domain-specific pathways implicated in schizophrenia, suggesting that those individual regions showing an independent significant association with $\mathrm{PRS}_{\mathrm{SZ}}$ may be more susceptible/resilient to the effect of $\mathrm{PRS}_{\mathrm{SZ}}$ beyond a latent factor of network integrity. We found several significant associations between PLEs-in particular regarding auditory hallucinations-and structural properties of the salience and DMN; with the right insular cortex largely mediating the association between auditory hallucinations and $\mathrm{PRS}_{\mathrm{SZ}}$. 
Impairments in white matter micro- and macrostructure are a common feature in schizophrenia with healthy relatives who are genetically at higher risk of developing schizophrenia also exhibiting impairments in $\mathrm{FA}^{58}$. Our results are in accordance to a previous study, reporting non-significant associations between a general factor of FA (including 27 major white matter tracts) and $\mathrm{PRS}_{\mathrm{SZ}}$ in a previous release of this data $(n=816)^{59}$, though the current approach offers greater brain regional fidelity and statistical power. Despite the apparent alterations in white matter structure in schizophrenia, only a small number of studies have reported significant associations between $\mathrm{PRS}_{\mathrm{SZ}}$ and white matter in healthy and clinical samples ${ }^{34,37,60}$. Significant reductions in global white matter volume in dizygotic twins discordant for schizophrenia have been reported while reductions in global grey matter were exclusively observed in schizophrenia $^{61}$. Thus, the null findings of white matter observed here may partly be due to the aggregation of genetic and environmental risk factors found in affected individuals and their relatives compared with healthy individuals.

\section{Psychotic-like experiences}

The reported prevalence of PLEs in this imaging sample $(4.75 \%)$ is similar to that in the whole UK Biobank sample $(5 \%)^{62}$, and with previous population studies ${ }^{63,64}$. We did not find any significant association between $\mathrm{PRS}_{\mathrm{SZ}}$ and total number of PLEs in the entire UKBiobank sample $(N=308,693)$, consistent with previous studies investigating this relationship in adolescence ${ }^{65,66}$. A recent UKBiobank study found a weak association between the presence of any PLEs and PRS $\mathrm{SZ}$ at a threshold of $p \leq 0.05$ (OR, 1.09; 95\% CI, 1.06-1.12; adjusted $R^{2}=0.001 ; p=$ $\left.2.96 \times 10^{-11}, N=500,000\right)$; however, as the authors noted, this association may be biased by the possibility that their analysis included sample overlap between UKBiobank and the training sets 9 .

The associations between PLEs and insular cortices are consistent with its involvement in interoception; this awareness of the body's internal state comprises emotional responses, complex cognitive states, and the sense of self ${ }^{67,68}$. Though our sample sizes were modest they are relatively large compared to the previous literature on PLEs and brain imaging in adults ${ }^{10-13,69}$ (for instance, studies on auditory hallucinations with samples of less than 30 participants compared to our sample of 51 experiencing auditory hallucinations and 134 for any PLEs). Moreover, our results are consistent with correlations between psychotic symptoms, activation, volume, and surface area of the insula in clinical ${ }^{70,71}$ and high-risk samples ${ }^{22}$; with hallucinations being associated with structural aspects of the insula ${ }^{72}$. Our mediation analyses extend these findings by showing the $\mathrm{CT}$ of the right insula largely mediating the association between $\mathrm{PRS}_{\mathrm{SZ}}$ and auditory hallucinations; in accordance with evidence suggesting that predominant right lateralization may discriminate auditory-verbal hallucinations from normal inner speech ${ }^{73}$. Impaired interoception has been associated with a loss of perception of the self, which may contribute to misattributions of agency in schizophrenia; indeed, higher frequency of prediction errors in sensory cues $^{74}$ and attribution of inner speech to that of others have been reported in the disorder ${ }^{75,76}$. Thus, we suggest that the observed insular differences may contribute to a higher susceptibility to commit external attribution errors in subjects experiencing PLEs. Overall, our results suggest that the salience network may play a key role in PLEs compared to the DMN and CEN. This is supported by studies showing that positive symptoms in schizophrenia are related to variations in the morphology, metabolism, and neural activity of the nodes of the salience network but not of DMN or $\mathrm{CEN}^{77}$.

The positive correlation between the GMV of the right pars orbitalis-ventral subregion of the inferior frontal gyrus (IFG) - and persecutory delusions is, however, at odds with previous studies reporting reduced GMV of the pars orbitalis in high risk individuals ${ }^{6,78}$ and negative associations between its volume and positive symptoms in clinical samples ${ }^{79}$; suggesting that involvement of the pars orbitalis may be limited to clinical populations. We also found that brain structure differences in the supramarginal gyrus were associated with delusions. This is in line with the role of the supramarginal gyrus in processing auditory inputs, especially language ${ }^{80}$, and the relation between lower volume and auditory hallucinations in schizophrenia $^{81}$. The supramarginal gyrus, here analyzed as a node in a salience network, is part of the somatosensory association cortex, which is involved in the interpretation of the postures and gestures of other people, including the empathic understanding of others. Disruption of the supramarginal gyrus has been linked to impaired empathy and people "projecting" emotions onto others, and such psychological processes have been implicated in delusions of persecution and reference since Freud $^{82}$. Intriguingly, we have previously shown fMRI over-activation and dysconnectivity of the intraparietal sulcus bordering the supramarginal gyrus in people at high risk of schizophrenia with sub-clinical (partial) delusions and hallucinations ${ }^{83,84}$. Although the relationship between structural and functional MRI is still not clear, it may be conceivable that over-activation of the supramarginal gyrus in people with such experiences could increase CT. In non-psychotic individuals, auditory hallucinations have been linked to abnormal brain connectivity within the DMN and with auditory cortices (sample of participants experiencing auditory hallucinations of $N=25$ and $N=29$, respectively ${ }^{10,12}$ ). The 
involvement of right supramarginal, insula, and IFG-all of them language-related areas-in PLEs has been extensively documented, indicating that those experiencing auditory hallucinations tend to show impaired speech perception $^{85}$ with an engagement of both speech production and reception circuitry ${ }^{86}$. Auditory hallucinations are commonly manifested as voices because the human auditory system is tuned to the natural priors of speech ${ }^{87}$ with its content possibly contributing to the formation of delusions by attributing meaning/agency to the experience, supported by our findings involving the supramarginal gyrus and delusions.

\section{Limitations}

We limited our analysis to those networks implicated in previous functional and structural studies, translating those nodes onto a common parcellation scheme. Given issues of validity and comparability across brain atlases, and the implications of this for underlying connectivity ${ }^{88-90}$, further research should aim to replicate this study by computing the networks at different levels of node granularity and with different structural properties. We applied a consistency-based thresholding method in an attempt to remove spurious white matter connections, exclusively preserving the top $30 \%$ that were most consistent across subjects. Though current evidence indicates that the human brain is likely to exhibit a fairly high degree of sparsity, principles of white matter connectivity in humans are not sufficiently comprehensive (when compared to the mouse ${ }^{91}$ ) to allow the confident implementation of detailed anatomical priors ${ }^{92}$; some connections may have therefore been pruned erroneously. We investigated whether neurostructural properties of brain networks are associated with PLEs. However, due to the still modest frequencies of PLEs in this sample, results should be interpreted cautiously. A previous study has indicated a potential "healthy volunteer" selection bias in this sample ${ }^{93}$, in particular, people with lower socioeconomic status, chronic illness, and smokers were under-represented $^{62}$. This suggests that this cohort may not be representative of the sampling population.

\section{Conclusions}

In a large sample of largely healthy participants from UKBiobank, a higher genetic liability for schizophrenia was associated with subtle neurostructural differences; in particular, a thinner cortex across the salience network. Beyond this global relationship, some independent paths emerged in each structural network. We also found significant associations between PLEs and the insula, supramarginal gyrus, and pars orbitalis; with the insula largely mediating the association between $\mathrm{PRS}_{\mathrm{SZ}}$ and auditory hallucinations. Our results in a large healthy sample support previous studies on aberrant activation of language-related areas and the externalization of inner speech phenomena in clinical samples. Studies with greater statistical power should validate the results of this research, which indicates that the study of the non-clinical phenotype may represent a valid approach to investigate the pathophysiology of the clinical phenotype and suggests a shared genetic aetiology between the clinical and non-clinical phenotype.

\section{Acknowledgements}

The authors thank the UK Biobank participants for their participation and the UK Biobank team for their work in collecting, processing, and disseminating these data for analysis. This research was conducted, using the UK Biobank Resource under approved project 16124 linked to 4844, in the Department of Psychiatry and Centre for Cognitive Ageing and Cognitive Epidemiology (CCACE) (http://www.ccace.ed.ac.uk) at The University of Edinburgh. S.R.C., J.W. M., M.E.B., C.R.B., I.J.D., and E.M.T.-D. were supported by a National Institutes of Health (NIH) research grant R01AG054628. E.M.T.-D. was also supported by National Institutes of Health $(\mathrm{NIH})$ research grant R01HD083613, and is a member of the Population Research Center at the University of Texas at Austin, which is supported by $\mathrm{NIH}$ center grant P2CHD042849. S.R.C. was supported by MRC grant MR/R024065/1. Also supported by the Spanish Ministry of Science, Innovation and Universities. Instituto de Salud Carlos III, co-financed by ERDF Funds from the European Commission, "A way of making Europe", CIBERSAM. Madrid Regional Government (B2017/BMD-3740 AGES-CM-2), European Union Structural Funds and European Union Seventh Framework Program and H2O2O Program; Fundación Familia Alonso, Fundación Alicia Koplowitz, and Fundación Mutua Madrileña.

\section{Author details \\ ${ }^{1}$ Division of Psychiatry, The University of Edinburgh, Edinburgh, UK. ${ }^{2}$ Department of Child and Adolescent Psychiatry, Institute of Psychiatry and Mental Health, Hospital General Universitario Gregorio Marañón, Madrid, Spain. ${ }^{3}$ Instituto de Investigación Sanitaria Gregorio Marañón (IiSGM), Madrid, Spain. ${ }^{4}$ Ciber del Area de Salud Mental (CIBERSAM), Madrid, Spain. ${ }^{5}$ MRC Centre for Reproductive Health, The University of Edinburgh, Edinburgh, UK. ${ }^{6}$ Centre for Clinical Brain Sciences, The University of Edinburgh, Edinburgh, UK. ${ }^{7}$ Centre for Cognitive Ageing and Cognitive Epidemiology, Department of Psychology, The University of Edinburgh, Edinburgh, UK. ${ }^{8}$ Department of Psychology, University of Texas at Austin, Austin, TX, USA. ${ }^{9}$ Scottish Imaging Network: A Platform for Scientific Excellence (SINAPSE), Edinburgh, UK. ${ }^{10}$ School of Medicine, Universidad Complutense, Madrid, Spain}

\section{Author contributions}

C.A. prepared the manuscript and was responsible for data preparation and statistical analyses. M.B.-C. assisted with network construction. S.R.C., S.M.L., and M.E.B. advised on the preparation of the manuscript, were involved in planning the study design and supervised the project. J.M.W. and E.M.T.-D. contributed to the analyses and advised on statistical procedures. J.J., I.J.D., H.C.W., C.A., and A.M.M. assisted with the interpretation of findings. J.G. provided all genetic data. All authors revised the manuscript.

\section{Conflict of interest}

C.A. has been a consultant to or has received honoraria or grants from Acadia, Angelini, Gedeon Richter, Janssen Cilag, Lundbeck, Otsuka, Roche, Sage, Servier, Shire, Schering Plough, Sumitomo Dainippon Pharma, Sunovion, and Takeda. In the last 3 years, S.M.L. has received research support from Janssen, and personal fees from Janssen and Sunovion. A.M.M. has previously received financial support from Janssen and Lilly. A.M.M., H.C.W., and S.M.L. have previously received financial support from Pfizer (formerly Wyeth) in relation to imaging studies of people with schizophrenia and bipolar disorder. All other authors report no biomedical financial interests or potential conflicts of interest.

\section{Publisher's note}

Springer Nature remains neutral with regard to jurisdictional claims in published maps and institutional affiliations. 
Supplementary Information accompanies this paper at (https://doi.org/ 10.1038/s41398-020-0794-x).

Received: 22 October 2019 Revised: 11 March 2020 Accepted: 25 March 2020

Published online: 27 April 2020

\section{References}

1. Kelly, S. et al. Widespread white matter microstructural differences in schizophrenia across 4322 individuals: results from the ENIGMA Schizophrenia DTI Working Group. Mol. Psychiatry https://doi.org/10.1038/mp.2017.170 (2017).

2. Lawrie, S. M. \& Abukmeil, S. S. Brain abnormality in schizophrenia. A systematic and quantitative review of volumetric magnetic resonance imaging studies. Br. J. Psychiatry: J. Ment. Sci. 172, 110-120 (1998).

3. van Erp, T. G. M. et al. Cortical brain abnormalities in 4474 individuals with schizophrenia and 5098 control subjects via the Enhancing Neuro Imaging Genetics Through Meta Analysis (ENIGMA) Consortium. Biol. Psychiatry https:// doi.org/10.1016/j.biopsych.2018.04.023 (2018).

4. Wright, I. C. et al. Meta-analysis of regional brain volumes in schizophrenia. Am. J. Psychiatry 157, 16-25 (2000).

5. van Os, J., Linscott, R. J., Myin-Germeys, I., Delespaul, P. \& Krabbendam, L. A systematic review and meta-analysis of the psychosis continuum: evidence for a psychosis proneness-persistence-impairment model of psychotic disorder. Psychol. Med. 39, 179-195 (2009).

6. DeRosse, P. et al. Evidence from structural and diffusion tensor imaging for frontotemporal deficits in psychometric schizotypy. Schizophr. Bull. 41, 104-114 (2015).

7. Cannon, M. et al. Evidence for early-childhood, pan-developmental impairment specific to schizophreniform disorder: results from a longitudinal birth cohort. Arch. Gen. Psychiatry 59, 449-456 (2002).

8. Hanssen, M., Bak, M., Bijl, R., Vollebergh, W. \& van Os, J. The incidence and outcome of subclinical psychotic experiences in the general population. Br. J. Clin. Psychol. 44, 181-191 (2005).

9. Legge, S. E. et al. Association of genetic liability to psychotic experiences with neuropsychotic disorders and traits. JAMA Psychiatry https://doi.org/10.1001/ jamapsychiatry.2019.2508 (2019).

10. Diederen, K. M. J. et al. Aberrant resting-state connectivity in non-psychotic individuals with auditory hallucinations. Psychol. Med. 43, 1685-1696 (2013).

11. Barber, A. D., Lindquist, M. A., DeRosse, P. \& Karlsgodt, K. H. Dynamic functional connectivity states reflecting psychotic-like experiences. Biol. Psychiatry Cogn. Neurosci. Neuroimaging 3, 443-453 (2018).

12. van Lutterveld, R., Diederen, K. M. J., Otte, W. M. \& Sommer, I. E. Network analysis of auditory hallucinations in nonpsychotic individuals. Hum. Brain Mapp. 35, 1436-1445 (2014).

13. Orr, J. M., Turner, J. A. \& Mittal, V. A. Widespread brain dysconnectivity associated with psychotic-like experiences in the general population. Neurolmage 4, 343-351 (2014)

14. Satterthwaite, T. D. et al. Connectome-wide network analysis of youth with psychosis spectrum symptoms. Mol. Psychiatry 20, 1508-1515 (2015).

15. Kelleher, I. \& Cannon, M. Psychotic-like experiences in the general population: characterizing a high-risk group for psychosis. Psychol. Med. 41, 1-6 (2011).

16. Menon, V. Large-scale brain networks and psychopathology: a unifying triple network model. Trends Cogn. Sci. 15, 483-506 (2011).

17. Palaniyappan, L., Mallikarjun, P., Joseph, V., White, T. P. \& Liddle, P. F. Regional contraction of brain surface area involves three large-scale networks in schizophrenia. Schizophr. Res. 129, 163-168 (2011).

18. Menon, V. Salience Network. In Brain Mapping. 597-611 Elsevier: 2015.

19. Uddin, L. Q. Salience processing and insular cortical function and dysfunction. Nat. Rev. Neurosci. 16, 55-61 (2015)

20. Manoliu, A. et al. Aberrant dependence of default mode/central executive network interactions on anterior insular salience network activity in schizophrenia. Schizophr. Bull. 40, 428-437 (2014).

21. Orliac, F. et al. Links among resting-state default-mode network, salience network, and symptomatology in schizophrenia. Schizophr. Res. 148, 74-80 (2013).

22. Wotruba, D. et al. Aberrant coupling within and across the default mode, taskpositive, and salience network in subjects at risk for psychosis. Schizophr. Bull. 40, 1095-1104 (2014).
23. Kapur, S. Psychosis as a state of aberrant salience: a framework linking biology, phenomenology, and pharmacology in schizophrenia. Am. J. Psychiatry 160, 13-23 (2003)

24. Buckner, R. L., Andrews-Hanna, J. R. \& Schacter, D. L. The brain's default network. Ann. N. Y. Acad. Sci. 1124, 1-38 (2008).

25. Zhou, $Y$. et al. Functional disintegration in paranoid schizophrenia using resting-state fMRI. Schizophr. Res. 97, 194-205 (2007).

26. Garrity, A. G. et al. Aberrant 'default mode' functional connectivity in schizophrenia. Am. J. Psychiatry 164, 450-457 (2007).

27. Harrison, B. J., Yücel, M., Pujol, J. \& Pantelis, C. Task-induced deactivation of midline cortical regions in schizophrenia assessed with fMRI. Schizophr. Res. 91, 82-86 (2007).

28. Bressler, S. L. \& Menon, V. Large-scale brain networks in cognition: emerging methods and principles. Trends Cogn. Sci. 14, 277-290 (2010).

29. Hilker, R. et al. Heritability of schizophrenia and schizophrenia spectrum based on the Nationwide Danish Twin Register. Biol. Psychiatry 83, 492-498 (2018).

30. International Schizophrenia ConsortiumCommon polygenic variation contributes to risk of schizophrenia and bipolar disorder. Nature 460, 748-752 (2009).

31. Schizophrenia Working Group of the Psychiatric Genomics Consortium Biological insights from 108 schizophrenia-associated genetic loci. Nature $\mathbf{5 1 1}$, 421-427 (2014)

32. Van der Auwera, S. et al. Predicting brain structure in population-based samples with biologically informed genetic scores for schizophrenia. Am. J. Med. Genet., Part B: Neuropsychiatr. Genet. 174, 324-332 (2017).

33. Van der Auwera, S. et al. No association between polygenic risk for schizophrenia and brain volume in the general population. Biol. Psychiatry 78, e41-e42 (2015)

34. Alloza, C. et al. Polygenic risk score for schizophrenia and structural brain connectivity in older age: a longitudinal connectome and tractography study. Neurolmage https://doi.org/10.1016/j.neuroimage.2018.08.075 (2018).

35. Mclntosh, A. M. et al. Polygenic risk for schizophrenia is associated with cognitive change between childhood and old age. Biol. Psychiatry 73, 938-943 (2013).

36. Neilson, E. et al. Impact of polygenic risk for schizophrenia on cortical structure in UK Biobank. Biol. Psychiatry 86, 536-544 (2019).

37. Ritchie, S. J. et al. Risk and protective factors for structural brain ageing in the eighth decade of life. Brain Struct. Funct. 222, 3477-3490 (2017).

38. Cox, S. R. et al. Compensation or inhibitory failure? Testing hypotheses of agerelated right frontal lobe involvement in verbal memory ability using structural and diffusion MRI. Cortex 63, 4-15 (2015).

39. Hoffman, P. et al. Brain grey and white matter predictors of verbal ability traits in older age: The Lothian Birth Cohort 1936. Neurolmage 156, 394-402 (2017).

40. Kirkpatrick, B., Messias, E., Harvey, P. D., Fernandez-Egea, E. \& Bowie, C. R. Is schizophrenia a syndrome of accelerated aging? Schizophr. Bull. 34, 1024-1032 (2008).

41. Kochunov, P. et al. Testing the hypothesis of accelerated cerebral white matter aging in schizophrenia and major depression. Biol. Psychiatry 73, 482-491 (2013).

42. Hagenaars, S. P. et al. Shared genetic aetiology between cognitive functions and physical and mental health in UK Biobank $(N=112151)$ and 24 GWAS consortia. Mol. Psychiatry 21, 1624-1632 (2016).

43. Euesden, J., Lewis, C. M. \& O'Reilly, P. F. PRSice: Polygenic Risk Score software. Bioinformatics 31, 1466-1468 (2015).

44. Miller, K. L. et al. Multimodal population brain imaging in the UK Biobank prospective epidemiological study. Nat. Neurosci. 19, 1523-1536 (2016).

45. Menon, V. \& Uddin, L. Q. Saliency, switching, attention and control: a network model of insula function. Brain Struct. Funct. 214, 655-667 (2010).

46. Catani, M., Dell'acqua, F. \& Thiebaut de Schotten, M. A revised limbic system model for memory, emotion and behaviour. Neurosci. Biobehav. Rev. 37, 1724-1737 (2013)

47. Power, J. D. et al. Functional network organization of the human brain. Neuron 72, 665-678 (2011)

48. Sridharan, D., Levitin, D. J. \& Menon, V. A critical role for the right fronto-insular cortex in switching between central-executive and default-mode networks. Proc. Natl Acad. Sci. USA. 105, 12569-12574 (2008).

49. Desikan, R. S. et al. An automated labeling system for subdividing the human cerebral cortex on MRI scans into gyral based regions of interest. Neurolmage 31, 968-980 (2006). 
50. Roberts, J. A., Perry, A., Roberts, G., Mitchell, P. B. \& Breakspear, M. Consistencybased thresholding of the human connectome. Neurolmage 145, 118-129 (2017).

51. Tucker-Drob, E. M. How many pathways underlie socioeconomic differences in the development of cognition and achievement? Learn. Individ. Differ. 25, 12-20 (2013)

52. Jöreskog, K. G. \& Goldberger, A. S. Estimation of a model with multiple indicators and multiple causes of a single latent variable. J. Am. Stat. Assoc. 70, 631-639 (1975).

53. Rosseel, Y. lavaan: an R package for structural equation modeling. J. Stat. Softw. 48, 36 (2012).

54. Ho, D., Imai, K., King, G. \& Stuart, E. A. Matchlt: nonparametric preprocessing for parametric causal inference. J. Stat. Softw. 42, 1-28 (2011).

55. Allen, M., Poggiali, D., Whitaker, K., Marshall, T. R. \& Kievit, R. Raincloud plots: a multi-platform tool for robust data visualization. Wellcome Open Res. https:// doi.org/10.12688/wellcomeopenres.15191.1 (2018).

56. Nuevo, R., Os, J. V., Arango, C., Chatterji, S. \& Ayuso-Mateos, J. L. Evidence for the early clinical relevance of hallucinatory-delusional states in the general population. Acta Psychiatr. Scand. 127, 482-493 (2013).

57. Benjamini, Y. \& Hochberg, Y. Controlling the false discovery rate: a practical and powerful approach to multiple testing. J. R. Stat. Soc. Ser. B 57, 289-300 (1995).

58. Muñoz Maniega, S. et al. A diffusion tensor MRI study of white matter integrity in subjects at high genetic risk of schizophrenia. Schizophr. Res. 106, 132-139 (2008).

59. Reus, L. M. et al. Association of polygenic risk for major psychiatric illness with subcortical volumes and white matter integrity in UK Biobank. Sci. Rep. 7, 42140 (2017).

60. Terwisscha van Scheltinga, A. et al. Genetic schizophrenia risk variants jointly modulate total brain and white matter volume. Biol. Psychiatry 73, 525-531 (2013).

61. Hulshoff Pol, H. E. et al. Gray and white matter volume abnormalities in monozygotic and same-gender dizygotic twins discordant for schizophrenia. Biol. Psychiatry 55, 126-130 (2004).

62. Davis, K. A. S. et al. Indicators of mental disorders in UK Biobank-a comparison of approaches. Int. J. Methods Psychiatr. Res. 28, e1796 (2019).

63. McGrath, J. J. et al. Psychotic experiences in the general population: a crossnational analysis based on 31,261 respondents from 18 countries. JAMA Psychiatry 72, 697-705 (2015).

64. Nuevo, R. et al. The continuum of psychotic symptoms in the general population: a cross-national study. Schizophr. Bull. 38, 475-485 (2012).

65. Jones, H. J. et al. Phenotypic manifestation of genetic risk for schizophrenia during adolescence in the general population. JAMA Psychiatry 73, 221-228 (2016).

66. Zammit, S. et al. A population-based study of genetic variation and psychotic experiences in adolescents. Schizophr. Bull. 40, 1254-1262 (2014).

67. Critchley, H. D., Wiens, S., Rotshtein, P., Ohman, A. \& Dolan, R. J. Neural systems supporting interoceptive awareness. Nat. Neurosci. 7, 189-195 (2004).

68. Damasio, A. Mental self: the person within. Nature 423, 227 (2003).

69. Fonville, L. et al. Psychotic experiences, working memory, and the developing brain: a multimodal neuroimaging study. Cereb. Cortex $\mathbf{2 5}$ 4828-4838 (2015).

70. Crespo-Facorro, B. et al. Insular cortex abnormalities in schizophrenia: a structural magnetic resonance imaging study of first-episode patients. Schizophr. Res. 46, 35-43 (2000).

71. Díaz-Caneja, C. M. et al. Neuroanatomical deficits shared by youth with autism spectrum disorders and psychotic disorders. Hum. Brain Mapp. 40, 1643-1653 (2019).
72. Crespo-Facorro, B. et al. Insular cortex morphometry in first-episode schizophrenia-spectrum patients: Diagnostic specificity and clinical correlations. J. Psychiatr. Res. 44, 314-320 (2010).

73. Sommer, I. E. C. et al. Auditory verbal hallucinations predominantly activate the right inferior frontal area. Brain 131, 3169-3177 (2008).

74. Synofzik, M., Thier, P., Leube, D. T., Schlotterbeck, P. \& Lindner, A. Misattributions of agency in schizophrenia are based on imprecise predictions about the sensory consequences of one's actions. Brain 133, 262-271 (2010).

75. Johns, L. C. et al. Verbal self-monitoring and auditory verbal hallucinations in patients with schizophrenia. Psychol. Med. 31, 705-715 (2001).

76. Costafreda, S. G., Brébion, G., Allen, P., McGuire, P. K. \& Fu, C. H. Y. Affective modulation of external misattribution bias in source monitoring in schizophrenia. Psychol. Med. 38, 821-824 (2008).

77. Supekar, K., Cai, W., Krishnadas, R., Palaniyappan, L. \& Menon, V. Dysregulated brain dynamics in a triple-network saliency model of schizophrenia and its relation to psychosis. Biol. Psychiatry 85, 60-69 (2019).

78. Francis, A. N. et al. Alterations in brain structures underlying language function in young adults at high familial risk for schizophrenia. Schizophr. Res. 141, 65-71 (2012).

79. Padmanabhan, J. L. et al. Correlations between brain structure and symptom dimensions of psychosis in schizophrenia, schizoaffective, and psychotic bipolar i disorders. Schizophr. Bull. 41, 154-162 (2015).

80. Paulesu, E., Frith, C. D. \& Frackowiak, R. S. The neural correlates of the verbal component of working memory. Nature 362, 342-345 (1993).

81. Gaser, C., Nenadic, I., Volz, H.-P., Büchel, C. \& Sauer, H. Neuroanatomy of 'hearing voices': a frontotemporal brain structural abnormality associated with auditory hallucinations in schizophrenia. Cereb. Cortex 14, 91-96 (2004).

82. Frith C. D. The Cognitive Neuropsychology of Schizophrenia. (Lawrence Erlbaum Associates, Inc:: 1992)

83. Whalley, H. C. et al. Functional disconnectivity in subjects at high genetic risk of schizophrenia. Brain 128, 2097-2108 (2005).

84. Whalley, H. C. et al. fMRI correlates of state and trait effects in subjects at genetically enhanced risk of schizophrenia. Brain 127, 478-490 (2004).

85. Hoffman, R. E., Rapaport, J., Mazure, C. M. \& Quinlan, D. M. Selective speech perception alterations in schizophrenic patients reporting hallucinated 'voices'. Am. J. Psychiatry 156, 393-399 (1999).

86. Zmigrod, L., Garrison, J. R., Carr, J. \& Simons, J. S. The neural mechanisms of hallucinations: a quantitative meta-analysis of neuroimaging studies. Neurosci. Biobehav. Rev. 69, 113-123 (2016).

87. Corlett, P. R. et al. Hallucinations and strong priors. Trends Cogn. Sci. 23 114-127 (2019).

88. Bohland, J. W., Bokil, H., Allen, C. B. \& Mitra, P. P. The brain atlas concordance problem: quantitative comparison of anatomical parcellations. PLOS ONE 4, e7200 (2009).

89. Cox, S. R. et al. A systematic review of brain frontal lobe parcellation techniques in magnetic resonance imaging. Brain Struct. Funct. 219, 1-22 (2014).

90. de Reus, M. A. \& van den Heuvel, M. P. The parcellation-based connectome: limitations and extensions. Neurolmage 80, 397-404 (2013).

91. Goulas, A., Uylings, H. B. M. \& Hilgetag, C. C. Principles of ipsilateral and contralateral cortico-cortical connectivity in the mouse. Brain Struct. Funct. 222 1281-1295 (2017)

92. Buchanan, C. R. et al. The effect of network thresholding and weighting on structural brain networks in the UK Biobank. bioRxiv https://doi.org/10.1101/ 649418 (2019).

93. Fry, A. et al. Comparison of sociodemographic and health-related characteristics of UK Biobank participants with those of the general population. Am. J. Epidemiol. 186, 1026-1034 (2017). 